SUPPORTING MATERIAL

\title{
Superacidic Activation of Quinoline and Isoquinoline; Their Reactions with Cyclohexane and Benzene
}

Konstantin Yu. Koltunov,* G.K. Surya Prakash, Golam Rasul, and George A. Olah

Table of contents:

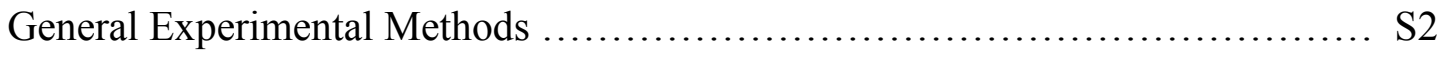

Cartesian coordinates of the $\mathrm{B} 3 \mathrm{LYP} / 6-31 \mathrm{G}^{*}$ optimized geometries and energies at the B3LYP/6-31G*//B3LYP/6-31G* level (in -Hartree) of 1a - e and 2a - e ..S2-S6

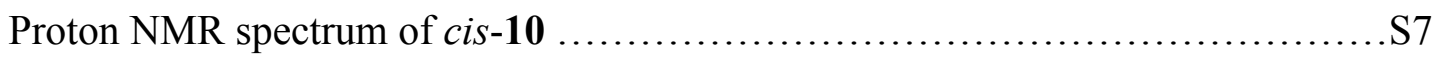

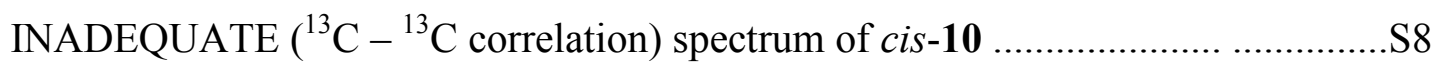

Proton NMR spectrum of mixture of cis/trans-10 (enriched with trans-10) ........ S9

Carbon-13 NMR spectrum of mixture of cis/trans-10 (enriched with trans-10) ... S10

Proton NMR spectrum of mixture of cis/trans-12 ..........................S11

Carbon-13 NMR spectrum of mixture of cis/trans-12 ....................... S12 


\section{General Experimental Methods}

${ }^{1} \mathrm{H}$ and ${ }^{13} \mathrm{C}$ NMR spectra were recorded on a $300 \mathrm{MHz}$ superconducting NMR spectrometer. The INADEQUATE $\left({ }^{13} \mathrm{C}-{ }^{13} \mathrm{C}\right.$ correlation) experiment is performed on a $500 \mathrm{MHz}$ NMR spectrometer. Trifluoromethanesulfonic and fluorosulfonic acids, sulfuryl fluoride chloride, aluminum halides and compounds $\mathbf{1}$ and $\mathbf{2}$ were purchased from chemical suppliers and used as received. Antimony pentafluoride was distilled under argon before use.

Cartesian coordinates of the $\mathrm{B} 3 \mathrm{LYP} / 6-31 \mathrm{G}^{*}$ optimized geometries and energies at the B3LYP/6-31G*//B3LYP/6-31G* level (in -Hartree, given in parentheses) of 1a - e and

$$
\begin{aligned}
& \mathbf{2 a}-\mathbf{e} \\
& \text { 1a (402.46606) } \\
& \mathrm{N}-1.2494 \quad-1.3244 \quad 0.0000 \\
& \begin{array}{llll}
\text { C } & -2.4464 & 0.7230 & -0.0000
\end{array} \\
& \begin{array}{llll}
\text { C } & -1.2517 & 1.4280 & -0.0000
\end{array} \\
& \begin{array}{llll}
\mathrm{C} & -0.0224 & 0.7313 & 0.0000
\end{array}
\end{aligned}
$$

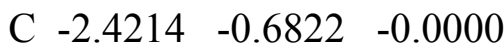

$$
\begin{aligned}
& \begin{array}{llll}
\text { C } & -0.0363 & -0.6751 & 0.0000
\end{array} \\
& \begin{array}{llll}
\text { C } & 1.2977 & 1.4256 & 0.0000
\end{array} \\
& \begin{array}{llll}
\text { C } & 2.4961 & 0.5796 & -0.0001
\end{array} \\
& \begin{array}{llll}
\text { C } & 2.4273 & -0.8095 & -0.0000
\end{array} \\
& \begin{array}{llll}
\text { C } & 1.1786 & -1.4283 & 0.0000
\end{array} \\
& \begin{array}{llll}
\mathrm{H} & -1.2621 & 2.5154 & -0.0000
\end{array} \\
& \text { H }-3.4049 \quad 1.2329-0.0000 \\
& \begin{array}{llll}
\mathrm{H} & -3.3204 & -1.2916 & 0.0000
\end{array} \\
& \begin{array}{llll}
\mathrm{H} & 1.1279 & -2.5170 & -0.0000
\end{array} \\
& \mathrm{H}-1.2604 \quad-2.3466 \quad 0.0000 \\
& \begin{array}{llll}
\mathrm{H} & 1.3710 & 2.1291 & 0.8564
\end{array} \\
& \begin{array}{llll}
\mathrm{H} & 3.4649 & 1.0772 & -0.0001
\end{array} \\
& \begin{array}{llll}
\mathrm{H} & 3.3303 & -1.4126 & -0.0000
\end{array} \\
& \begin{array}{llll}
\mathrm{H} & 1.3709 & 2.1295 & -0.8559
\end{array} \\
& \text { 1b (402.46155) } \\
& \begin{array}{llll}
\mathrm{N} & 1.2242 & -1.3253 & 0.0000
\end{array} \\
& \begin{array}{llll}
\text { C } & 2.4741 & 0.7169 & -0.0000
\end{array} \\
& \begin{array}{llll}
\text { C } & 1.2916 & 1.4337 & -0.0000
\end{array} \\
& \begin{array}{llll}
\text { C } & 0.0345 & 0.7493 & -0.0000
\end{array} \\
& \begin{array}{llll}
\text { C } & 2.4102 & -0.6802 & -0.0000
\end{array} \\
& \begin{array}{llll}
\text { C } & 0.0107 & -0.6930 & 0.0000
\end{array}
\end{aligned}
$$




$\begin{array}{lrrr}\text { C } & -1.1866 & 1.4198 & -0.0000 \\ \text { C } & -2.4532 & 0.6921 & 0.0000 \\ \text { C } & -2.3880 & -0.7827 & 0.0000 \\ \text { C } & -1.1934 & -1.4469 & 0.0000 \\ \text { H } & -3.0802 & 1.0564 & 0.8460 \\ \text { H } & 3.4430 & 1.2057 & -0.0000 \\ \text { H } & 3.2970 & -1.3087 & -0.0000 \\ \text { H } & -1.1565 & -2.5333 & -0.0000 \\ \text { H } & 1.2371 & -2.3476 & 0.0000 \\ \text { H } & -1.2107 & 2.5087 & -0.0000 \\ \text { H } & -3.0803 & 1.0565 & -0.8459 \\ \text { H } & -3.3241 & -1.3358 & 0.0000 \\ \text { H } & 1.3058 & 2.5213 & -0.0000 \\ & & & \\ \text { 1c } & (402.45459) & \\ \text { N } & -1.2598 & -1.3356 & 0.0000 \\ \text { C } & -2.4506 & 0.7282 & -0.0000 \\ \text { C } & -1.2750 & 1.4379 & 0.0000 \\ \text { C } & -0.0138 & 0.7531 & 0.0000 \\ \text { C } & -2.4161 & -0.6958 & 0.0000 \\ \text { C } & -0.0232 & -0.6864 & 0.0000 \\ \text { C } & 1.2195 & 1.4616 & -0.0000 \\ \text { C } & 2.4076 & 0.7749 & -0.0000 \\ \text { C } & 2.4381 & -0.6964 & -0.0000 \\ \text { C } & 1.1579 & -1.4044 & -0.0000 \\ \text { H } & -1.2882 & 2.5248 & -0.0000 \\ \text { H } & -3.4166 & 1.2243 & -0.0001 \\ \text { H } & -3.3204 & -1.2991 & -0.0000 \\ \text { H } & 1.1636 & -2.4936 & -0.0001 \\ \text { H } & -1.2688 & -2.3580 & 0.0000 \\ \text { H } & 1.2030 & 2.5475 & -0.0000 \\ \text { H } & 3.3563 & 1.3064 & -0.0000 \\ \text { H } & 3.0617 & -1.0698 & 0.8451 \\ \text { H } & 3.0620 & -1.0699 & -0.8448 \\ & & & \\ \text { 1d } & (402.46805) & \\ \text { N } & -1.2087 & -1.3130 & 0.0000 \\ \text { C } & -2.4725 & 0.7062 & 0.0000 \\ \text { C } & -1.2855 & 1.4299 & 0.0000 \\ \text { C } & -0.0405 & 0.7485 & 0.0000 \\ \text { C } & -2.4112 & -0.6834 & 0.0000 \\ \text { C } & -0.0224 & -0.6614 & 0.0000 \\ \text { C } & 1.2150 & 1.4437 & -0.0000 \\ \text { C } & 2.4518 & 0.7908 & -0.0001 \\ \text { C } & 2.4841 & -0.5910 & -0.0000 \\ \text { C } & 1.2609 & -1.4212 & 0.0001 \\ \text { H } & 1.3149 & -2.1102 & 0.8668\end{array}$




\begin{tabular}{|c|c|c|}
\hline H -3.4396 & 1.1986 & 0.0000 \\
\hline H -3.2893 & -1.3221 & 0.0000 \\
\hline Н 1.3148 & -2.1105 & -0.8664 \\
\hline H -1.2121 & -2.3355 & 0.0000 \\
\hline Н 1.1924 & 2.5328 & -0.0000 \\
\hline H 3.3715 & 1.3680 & -0.0001 \\
\hline Н 3.4361 & -1.1196 & -0.0001 \\
\hline H -1.3067 & 2.5171 & 0.0000 \\
\hline \multicolumn{3}{|c|}{$1 \mathbf{e}(402.45328)$} \\
\hline N 1.1987 & -1.3494 & -0.0000 \\
\hline C 2.4261 & 0.7355 & 0.0000 \\
\hline C 1.1225 & 1.4351 & -0.0000 \\
\hline C -0.0721 & 0.7491 & 0.0000 \\
\hline C 2.3493 & -0.7407 & 0.0000 \\
\hline C -0.0583 & -0.7004 & -0.0000 \\
\hline C -1.3403 & 1.4198 & 0.0000 \\
\hline C -2.5130 & 0.6856 & 0.0000 \\
\hline C -2.4549 & -0.7214 & -0.0000 \\
\hline C -1.2279 & -1.4258 & -0.0000 \\
\hline Н 1.1343 & & \\
\hline $\mathrm{H} \quad 3.04^{\prime}$ & 1.0704 & 0.8572 \\
\hline H 3.2500 & -1.3523 & 0.0001 \\
\hline H -1.2299 & -2.5129 & -0.0000 \\
\hline Н 1.1884 & -2.3743 & 0.0000 \\
\hline H - -1.3608 & 2.5065 & 0.0001 \\
\hline H -3.4761 & 1.1865 & 0.0000 \\
\hline H -3.3796 & -1.2935 & -0.0000 \\
\hline Н 3.0472 & 1.0704 & -0.8571 \\
\hline \multicolumn{3}{|c|}{ 2a $(402.47280)$} \\
\hline $\mathrm{N}-2.3917$ & -0.6535 & -0.0000 \\
\hline C -2.4143 & 0.7096 & -0.0000 \\
\hline C -1.2267 & 1.4169 & 0.0000 \\
\hline C -1.2618 & -1.3708 & -0.0000 \\
\hline C -0.0075 & 0.7216 & 0.0000 \\
\hline C -0.0256 & -0.7012 & -0.0000 \\
\hline C 1.3111 & 1.4238 & 0.0000 \\
\hline C 2.5121 & 0.5719 & 0.0000 \\
\hline C 2.4529 & -0.8141 & -0.0000 \\
\hline C 1.2061 & -1.4390 & -0.0000 \\
\hline Н 1.3804 & 2.1242 & -0.8576 \\
\hline Н -3.3929 & 1.1790 & -0.0000 \\
\hline H -3.2854 & -1.1512 & -0.0000 \\
\hline Н 1.1602 & -2.5272 & -0.0000 \\
\hline H -1.3580 & -2.4526 & -0.0000 \\
\hline Н 1.3804 & 2.1241 & 0.8577 \\
\hline
\end{tabular}




\begin{tabular}{cccc} 
H & 3.4788 & 1.0740 & 0.0000 \\
H & 3.3609 & -1.4094 & -0.0000 \\
H & -1.2612 & 2.5023 & 0.0000 \\
2b & \multicolumn{3}{c}{$(402.45256)$} \\
N & -2.3770 & 0.6552 & 0.0000 \\
C & -2.4384 & -0.7168 & 0.0000 \\
C & -1.2654 & -1.4289 & 0.0000 \\
C & -1.2580 & 1.3720 & -0.0000 \\
C & -0.0171 & -0.7343 & -0.0000 \\
C & 0.0015 & 0.7097 & -0.0000 \\
C & 1.2116 & -1.4113 & -0.0001 \\
C & 2.4684 & -0.6771 & 0.0000 \\
C & 2.4160 & 0.7946 & 0.0000 \\
C & 1.2156 & 1.4566 & -0.0000 \\
H & -1.2964 & -2.5144 & 0.0000 \\
H & -3.4280 & -1.1598 & 0.0001 \\
H & -3.2665 & 1.1638 & 0.0000 \\
H & 1.1787 & 2.5422 & -0.0001 \\
H & -1.3554 & 2.4537 & -0.0000 \\
H & 1.2407 & -2.5000 & -0.0001 \\
H & 3.1029 & -1.0505 & -0.8394 \\
H & 3.3550 & 1.3422 & 0.0002 \\
H & 3.1027 & -1.0505 & 0.8396 \\
& & & \\
2c & $4402.46108)$ & & \\
N & -2.3891 & -0.6719 & -0.0000 \\
C & -2.4179 & 0.7132 & 0.0000 \\
C & -1.2506 & 1.4275 & 0.0000 \\
C & -1.2682 & -1.3760 & -0.0000 \\
C & 0.0032 & 0.7533 & 0.0000 \\
C & -0.0170 & -0.6985 & -0.0000 \\
C & 1.2419 & 1.4565 & -0.0000 \\
C & 2.4233 & 0.7655 & -0.0000 \\
C & 2.4595 & -0.7119 & 0.0000 \\
C & 1.1783 & -1.4126 & -0.0000 \\
H & 3.0782 & -1.0922 & -0.8453 \\
H & -3.4040 & 1.1661 & 0.0000 \\
H & -3.2836 & -1.1689 & -0.0000 \\
H & 1.1788 & -2.5019 & -0.0000 \\
H & -1.3519 & -2.4597 & -0.0000 \\
H & 1.2344 & 2.5426 & -0.0000 \\
H & 3.3732 & 1.2947 & -0.0000 \\
H & 3.0781 & -1.0921 & 0.8455 \\
H & -1.2948 & 2.5123 & 0.0000 \\
& & & \\
2d & $(402.46802)$ & \\
& & &
\end{tabular}




$\begin{array}{lrrr}\text { N } & -2.3788 & -0.6492 & -0.0001 \\ \text { C } & -2.4346 & 0.7058 & -0.0000 \\ \text { C } & -1.2523 & 1.4232 & 0.0001 \\ \text { C } & -1.2368 & -1.3621 & -0.0001 \\ \text { C } & -0.0242 & 0.7260 & 0.0001 \\ \text { C } & -0.0122 & -0.6926 & 0.0000 \\ \text { C } & 1.2356 & 1.4373 & 0.0000 \\ \text { C } & 2.4663 & 0.7903 & -0.0001 \\ \text { C } & 2.5004 & -0.6023 & -0.0001 \\ \text { C } & 1.2887 & -1.4303 & 0.0001 \\ \text { H } & 1.3576 & -2.1295 & 0.8592 \\ \text { H } & -3.4197 & 1.1602 & -0.0000 \\ \text { H } & -3.2631 & -1.1641 & -0.0001 \\ \text { H } & 1.3575 & -2.1304 & -0.8581 \\ \text { H } & -1.3343 & -2.4434 & -0.0001 \\ \text { H } & 1.2079 & 2.5260 & 0.0001 \\ \text { H } & 3.3871 & 1.3661 & -0.0002 \\ \text { H } & 3.4589 & -1.1204 & -0.0002 \\ \text { H } & -1.2864 & 2.5084 & 0.0001 \\ & & & \\ \text { 2e } & (402.44677) & \\ \text { N } & -2.3257 & -0.7625 & -0.0001 \\ \text { C } & -2.4576 & 0.5439 & -0.0002 \\ \text { C } & -1.2853 & 1.4222 & 0.0002 \\ \text { C } & -1.0873 & -1.4133 & 0.0000 \\ \text { C } & 0.0540 & 0.7457 & 0.0001 \\ \text { C } & 0.0921 & -0.7118 & 0.0001 \\ \text { C } & 1.2449 & 1.4386 & -0.0001 \\ \text { C } & 2.4638 & 0.7256 & -0.0002 \\ \text { C } & 2.5189 & -0.6904 & -0.0000 \\ \text { C } & 1.3500 & -1.4133 & 0.0001 \\ \text { H } & -1.4007 & 2.1157 & 0.8593 \\ \text { H } & -3.4691 & 0.9491 & -0.0003 \\ \text { H } & -3.1662 & -1.3490 & -0.0003 \\ \text { H } & 1.3579 & -2.4999 & 0.0002 \\ \text { H } & -1.1414 & -2.4987 & 0.0000 \\ \text { H } & 1.2626 & 2.5249 & -0.0002 \\ \text { H } & 3.3950 & 1.2877 & -0.0003 \\ \text { H } & 3.4814 & -1.1923 & -0.0001 \\ \text { H } & -1.4005 & 2.1168 & -0.8580 \\ & & & \end{array}$




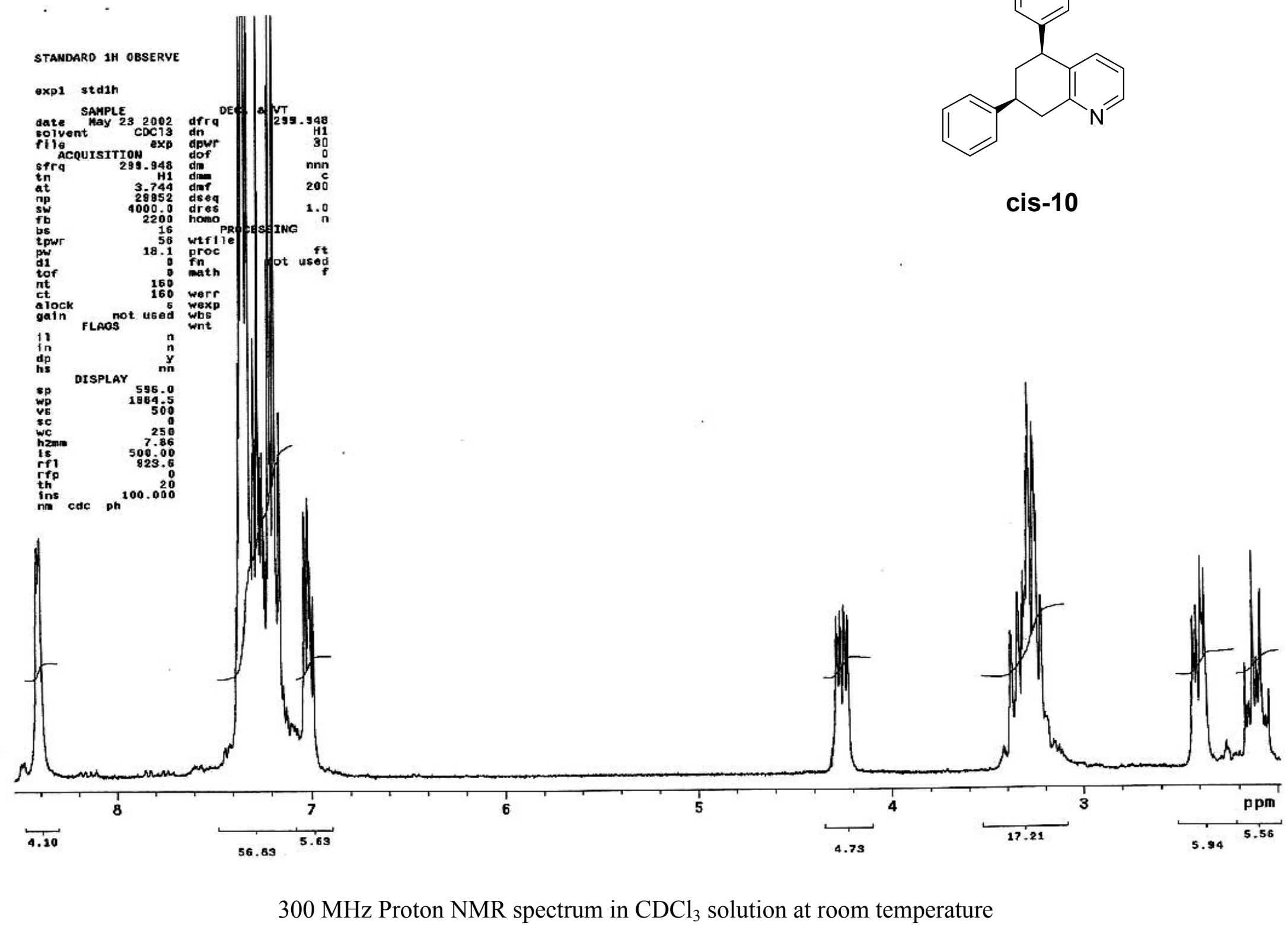




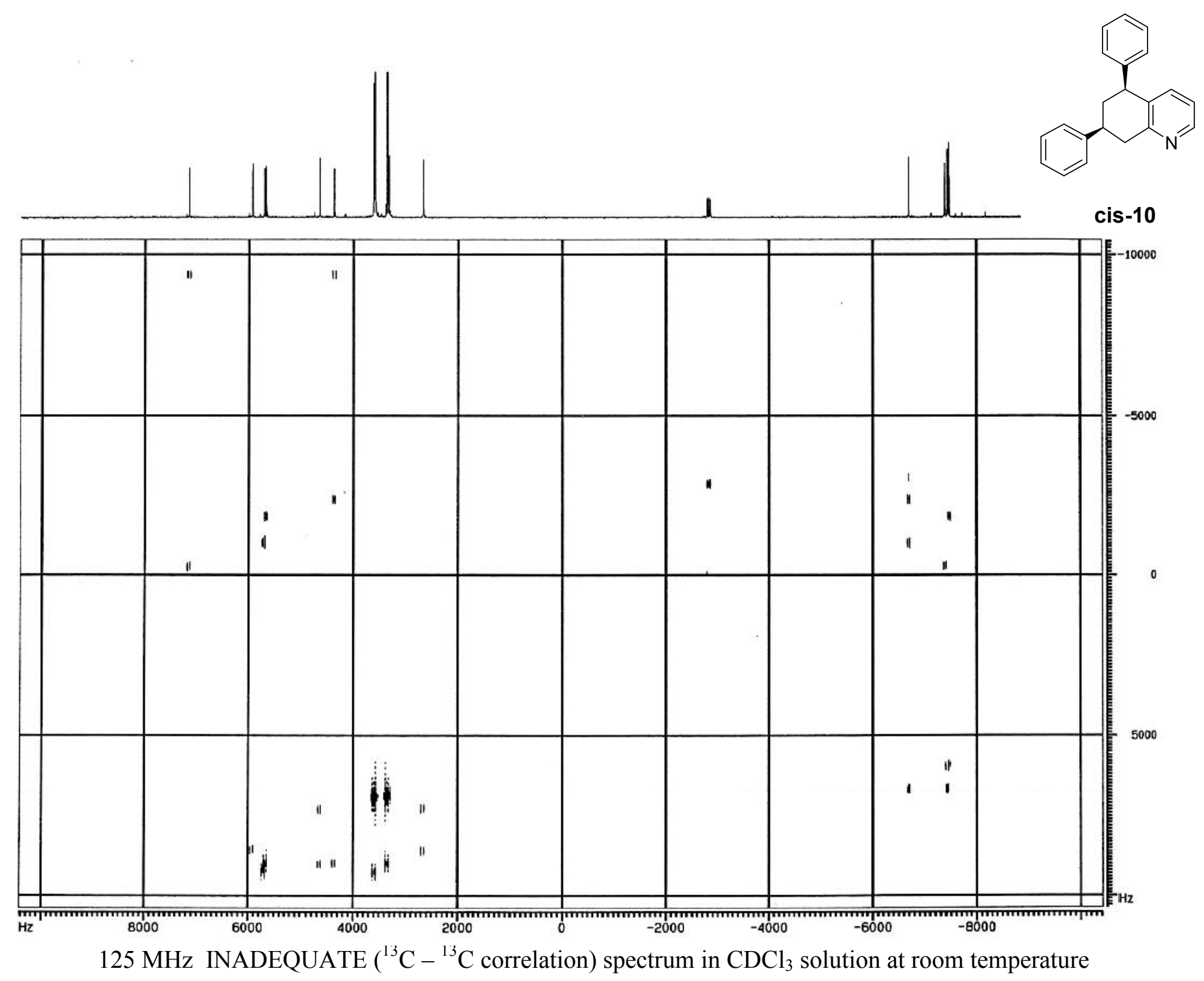




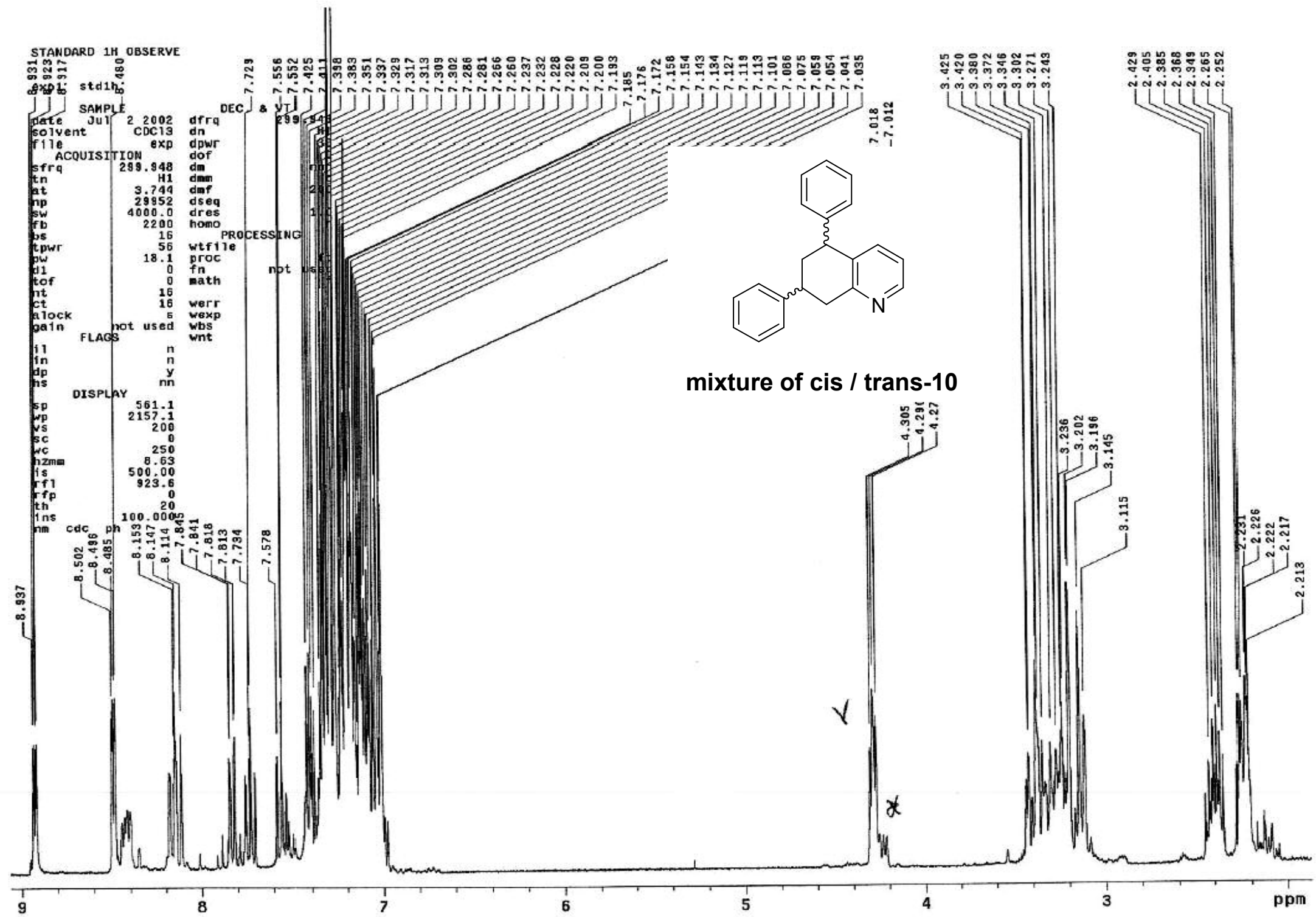

$300 \mathrm{MHz}$ Proton NMR spectrum of mixture of cis/trans-10 (enriched with trans-10 and containing impurity of $\mathbf{1}$ ) in $\mathrm{CDCl}_{3}$ 


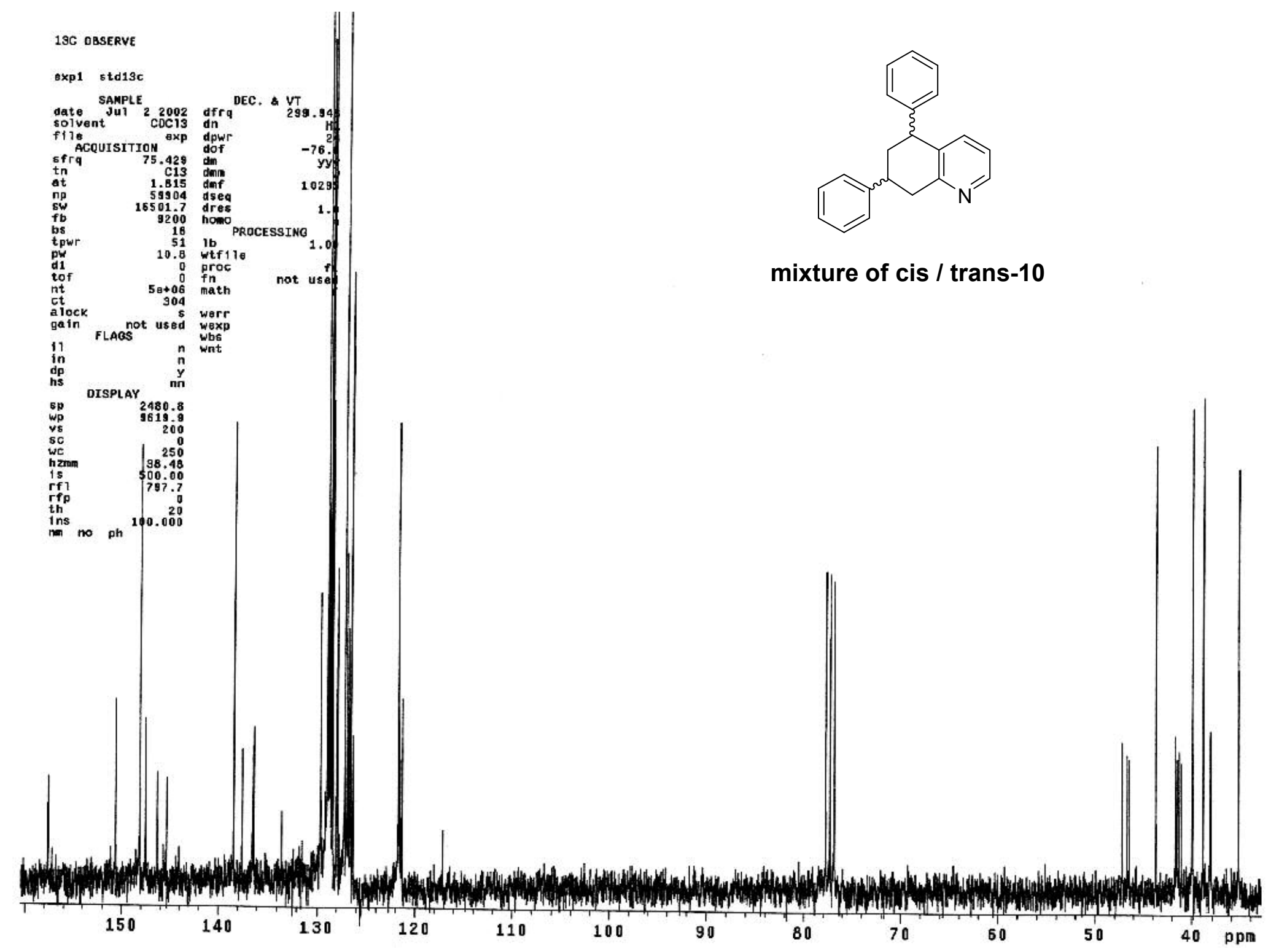

$75 \mathrm{MHz}$ Carbon-13 NMR spectrum of mixture of cis/trans-10 (enriched with trans-10) in $\mathrm{CDCl}_{3}$ solution at room temperature 


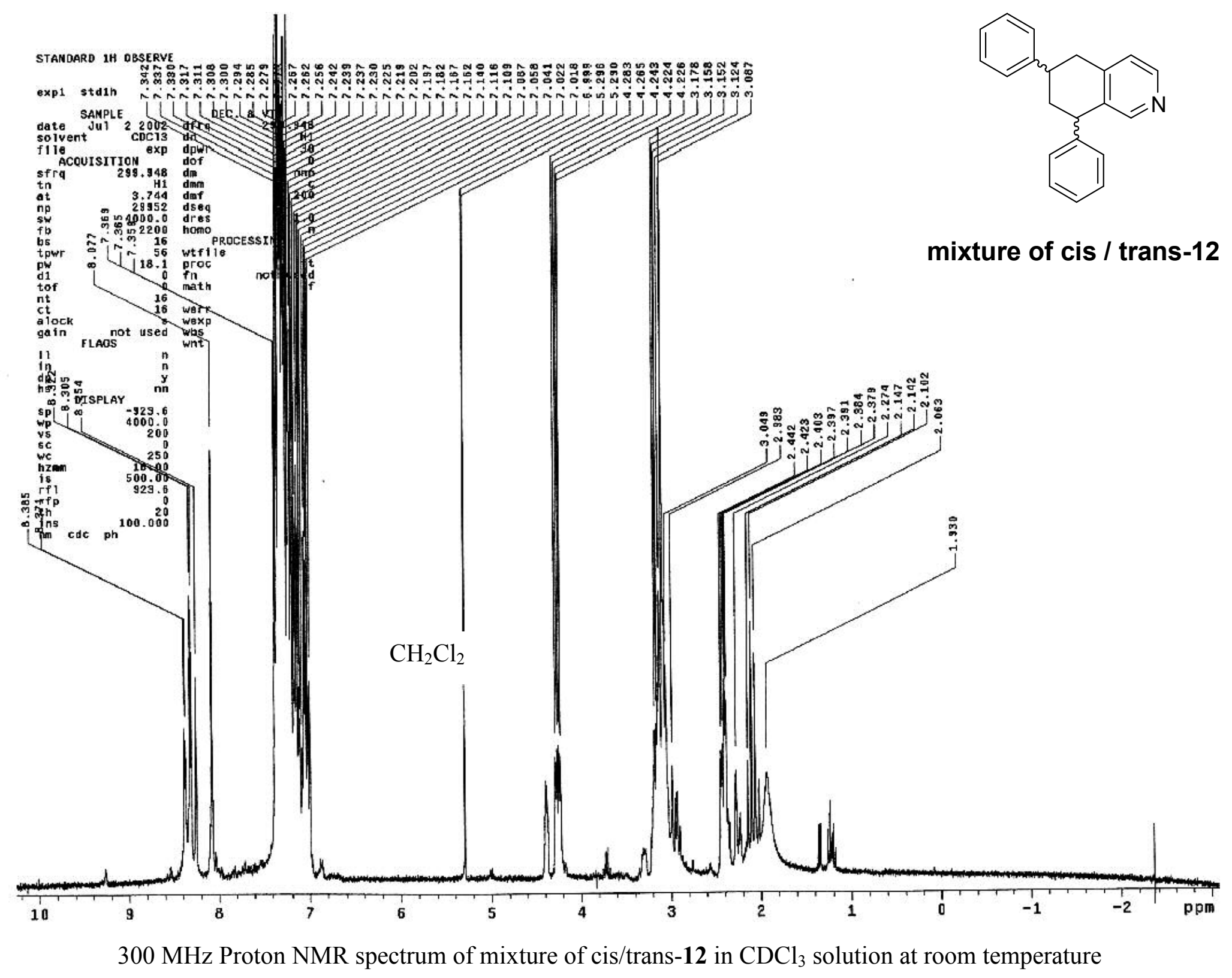




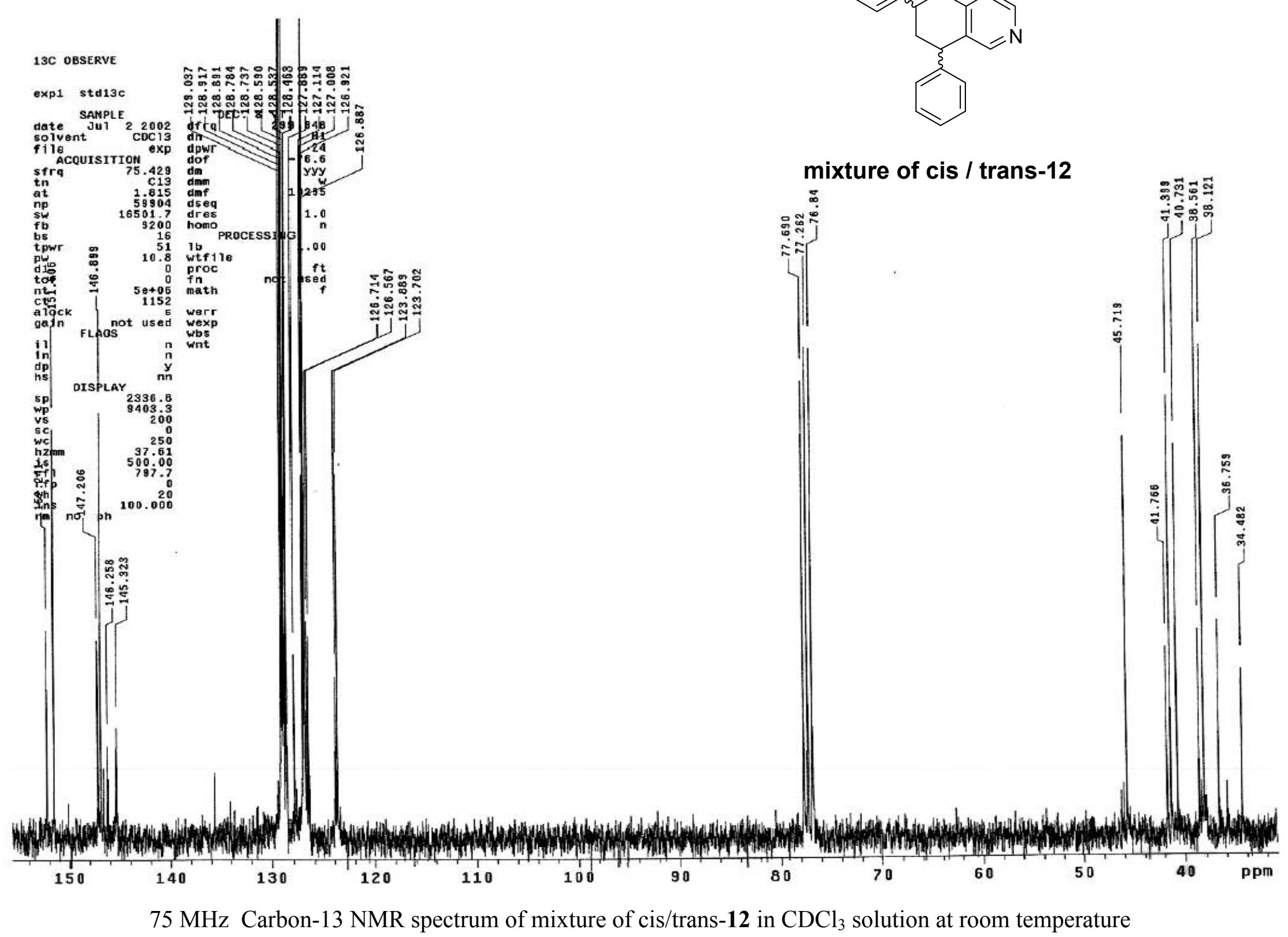

